\title{
New surgical technique for hallux valgus: a multiplanar chevron osteotomy
}

\author{
Volume 4 Issue I - 2016 \\ Jorge de las Heras Romero \\ University Hospital Reina Sofia, Spain
}

\begin{abstract}
Correspondence: Jorge de las Heras Romero, Traumatology Department, University Hospital Reina Sofia, Avda. Intendente Jorge Palacios, I, 30003 , Murcia, Spain, Tel 34968359000 Email jorgeheras@gmail.com
\end{abstract}

Received: December 13, 2015 | Published: January 08, 2016
Multiple techniques have been described in the literature, most of them specifically designed for treatment of non-degenerative Hallux Valgus.

In the case of arthritic deformity, current literature recommends first metatarsophalangeal joint (MTPJ) resection arthroplasty with or without joint implant, or arthrodesis, depending on individual cases.

The goal is to correct the deformity while the joint is excised therefore decreasing the pain, but these techniques have plenty of side-effects, namely recurrence of deformity, loss of flexion strength of first MTPJ causing first ray insufficiency, first MTPJ loss of movement, and so on.

The surgical procedure described in this paper is especially useful for Hallux Valgus associated to arthritic changes (although it has also been used by the author in non-arthritic cases, especially in patients with associated metatarsalgia, avoiding Weil osteotomies) and to date is the first described distal metatarsal osteotomy for this purpose.

It implies a correction of the deformity, decompression of the first MTPJ and lowering of the first metatarsal head, setting up a combination of Austin osteotomy. ${ }^{1}$ for Hallux Valgus with Weil osteotomy. ${ }^{2}$ for Hallux Rigidus, addressing both problems at the same time, so as to avoid all complications that come with other more aggressive techniques.

In this procedure a "V" shape osteotomy of the distal metatarsal is created, allowing the first metatarsal head to be shifted not only laterally, as in the original Austin Chevron osteotomy. ${ }^{1}$ technique, but also backwards and downwards.

In case of increased distal metatarsal articular angle (DMAA) there is an option of removing a wedge from the upper arm of the osteotomy twisting the metatarsal head medially, correcting this way the orientation of the MTPJ. ${ }^{3}$

Firstly, the initial cut of the osteotomy is made at 1 o'clock position from medial and distal to lateral and proximal, setting up a 20 degrees angle with a perpendicular line to the longitudinal axis of the metatarsal. The result of this cut is shortening and lateral displacement of the metatarsal head (Figure 1).

Secondly, the other osteotomy is performed at a 4 o'clock position with the direction of the cut from medial and dorsal to lateral and plantar, setting up a 20 degrees angle with, again, a perpendicular line to the longitudinal axis of the mentioned metatarsal. The result of this cut is lowering and lateral displacement of the metatarsal head (Figure 2).
Finally, in case of requiring a correction of the DMAA we can remove a wedge, with its base pointing medially, from the bone proximal to the first cut at the appropriate angle.

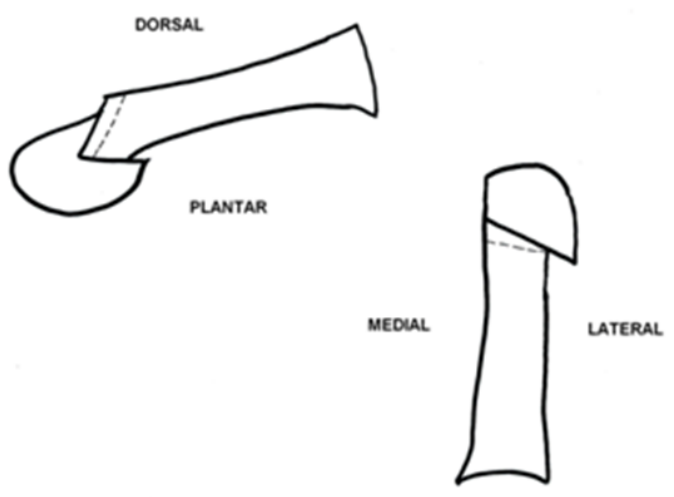

Figure I Multiplanar metatarsal osteotomy. Plantar, lateral and posterior displacement of the metatarsal head. In case of increased DMAA, a closing wedge osteotomy can be performed (dotted line).

To fix the deformity the author usually employ only 1 cannulated double threaded $3.5 \mathrm{~mm}$ screw as this osteotomy is very stable. It is designed to be place from superior and proximal to inferior and distal in the head direction with the tip of the screw located in the subchondral bone of the metatarsal head.

Before achieving this osteotomy a trans-articular adductor tenotomy, a cheilectomy and a buniectomy is usually performed, all through the same medial approach.

If the Hallux Abductus Angle is more than 30 degrees or the deformity is classified at least as moderate (grade 3) using the 
Manchester Scale. ${ }^{4}$ an Akin phalangeal osteotomy. ${ }^{2}$ is always added and fixed with a cannulated double threaded $2.5 \mathrm{~mm}$ screw across the osteotomy line (Figure $3 \& 4$ ).

At last, a realignment medial capsulorrhaphy is performed at the end of the procedure.
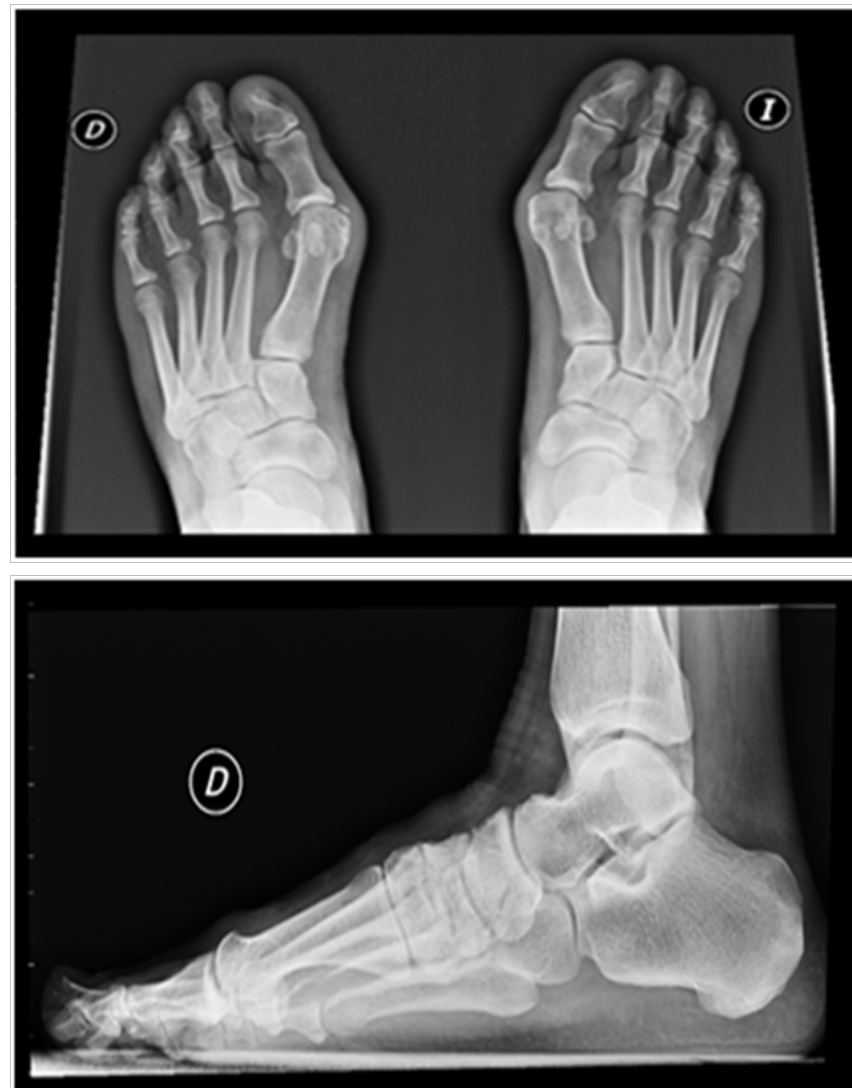

Figure 2 Preoperative Rx showing Hallux Valgus.

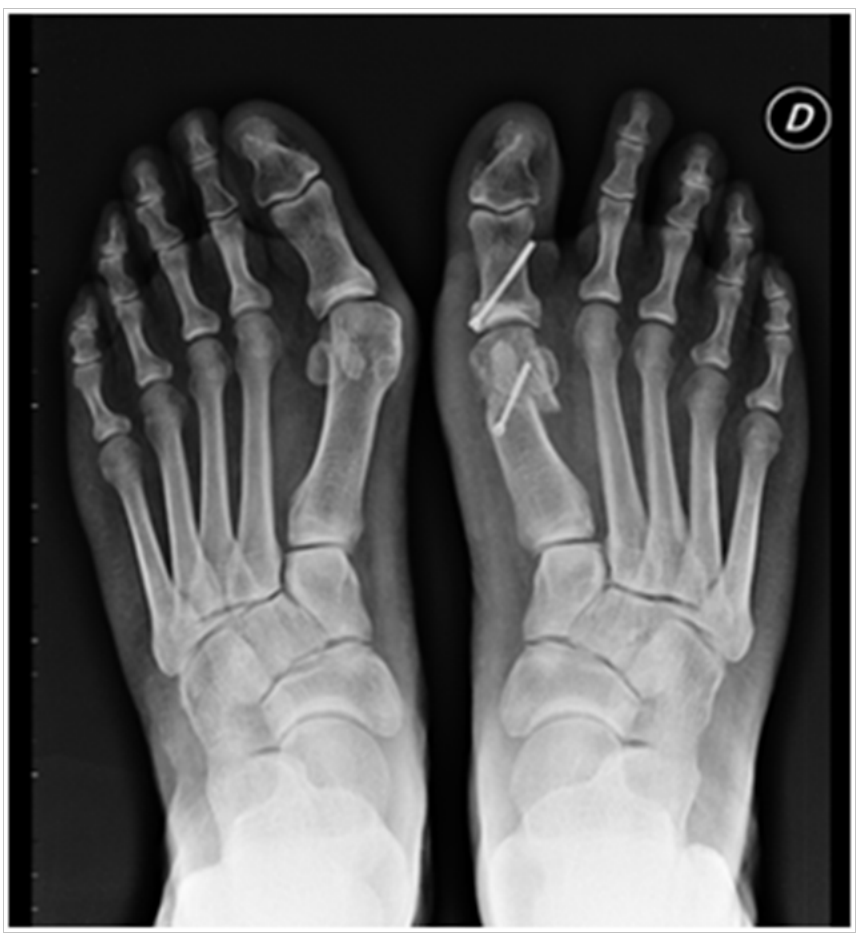

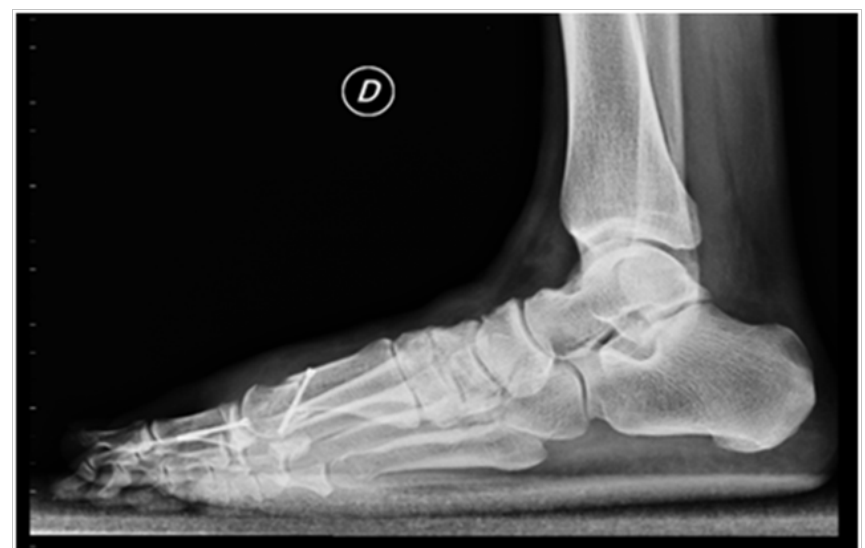

Figure 3 Postoperative $X$-rays 3 weeks after surgery.
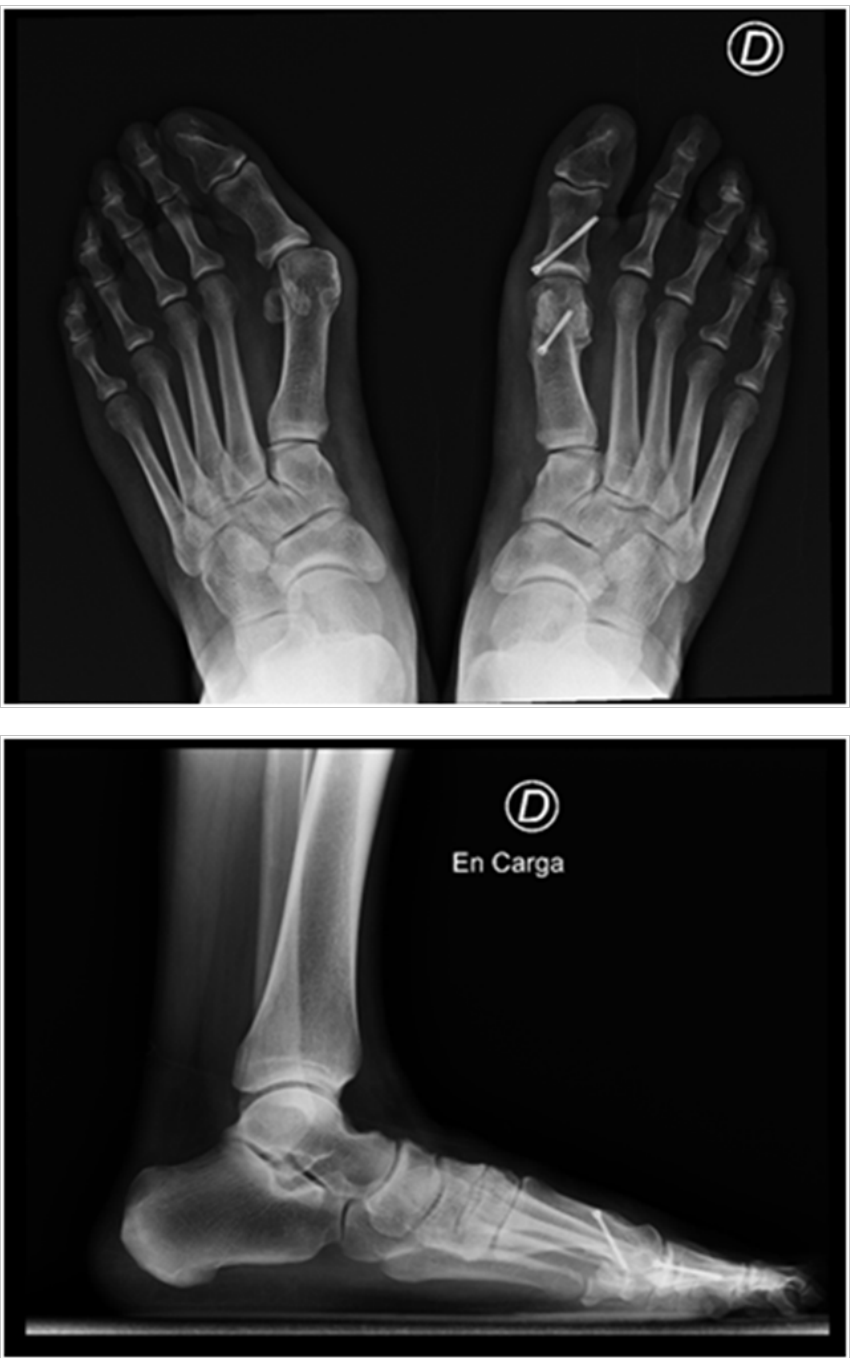

Figure 4 Postoperative $X$-rays 3 months after surgery.

\section{Ackowledgments}

None.

\section{Conflicts of interst}

None. 


\section{References}

1. Austin DW, Leventen EO A new osteotomy for hallux valgus: a horizontally directed "V" displacement osteotomy of the metatarsal head for hallux valgus and primus varus. Clin Ortbop. 1981:157:25-30.

2. Barouk LS Weil's metatarsal osteotomy in the treatment of metatarsalgia. Orthopade. 1996:25(4):338-344.
3. Corte-Real NM, Moreira RM Modified biplanar chevron osteotomy. Foot Ankle Int. 2009:30(12):1149-1153.

4. Menz HB, Munteanu SE Radiographic validation of the Manchester scale for the classification of hallux valgus deformity. Rheumatology. 2003:44(8):1061-1066. 